Anaesthesist 2022 $\cdot 71: 318-320$

https://doi.org/10.1007/s00101-022-01099-7

Angenommen: 25. Januar 2022

Online publiziert: 18. Februar 2022

( $)$ The Author(s), under exclusive licence to Springer Medizin Verlag GmbH, ein Teil von Springer Nature 2022

\section{Anteil an Geimpften in der Bevölkerung und Coronavirus Disease 2019(COVID-19)-Fälle - Limitationen und irreführende Schlussfolgerungen}

Julian Umlauf · Axel Rüdiger Heller

Klinik für Anästhesiologie und Intensivmedizin, Universitätsklinikum Augsburg, Augsburg, Deutschland
Originalpublikation

Subramanian SV, Kumar A (2021) Increases in COVID-19 are unrelated to levels of vaccination across 68 countries and 2947 counties in the United States. Eur J Epidemiol 36(12):1237-1240. https://doi.org/10.1007/ s10654-021-00808-7.

Hintergrund. Das Ziel des Artikels von Subramanian und Kumar ist es, zu überprüfen, ob es einen Zusammenhang zwischen dem prozentuellen Anteil an vollständig Geimpften und neu aufgetretenen Coronavirus Disease 2019(COVID-19)Fällen gibt. Die Analyse wurde mit Daten aus 68 Ländern weltweit und 2947 Landkreisen ("counties ${ }^{\prime \prime}$ ) in den USA vollzogen [1].

Methodik. Für die länderübergreifende Analyse wurden die Daten der Internetseite von Our World in Data (https:// ourworldindata.org/coronavirus), besucht am 03.09.2021, benutzt. Die Kriterien, welche zum Einschluss der 68 Länder führten, waren wie folgt: Verfügbarkeit von Daten über den Anteil an Zweitgeimpften in der Bevölkerung, Verfügbarkeit über neu aufgetretene COVID-19-Fälle, Verfügbarkeit von Bevölkerungsdaten und letzte Aktualisierung der Daten spätestens 3 Tage vor dem 03.09.2021.

Für die Analyse auf Landkreisebene wurden Daten des "White House COVID 19 Team" verwendet und am 02.09.2021 abgerufen (https://healthdata. gov/Health/COVID-19-Community-ProfileReport/gqxm-d9w9). Ausgeschlossen wurden Landkreise mit unvollständigen
Daten über den Anteil an vollständig Geimpften; dies führte zum Einschluss von 2947 Landkreisen. Der prozentuelle Anstieg an COVID-19-Fällen in den jeweiligen untersuchten Gebieten wurde basierend auf der Differenz zwischen den neu aufgetretenen Fällen in den letzten 7 Tagen und den neu aufgetretenen Fällen in den 7 Tagen davor berechnet. Der Artikel beinhaltet keine Informationen über eine statistische Methode.

Ergebnisse. Sowohl auf Ebene der Länder als auch auf Ebene der US-Landkreise konnte kein Zusammenhang zwischen prozentuellem Anteil der vollständig geimpften Bevölkerung und neu aufgetretenen COVID-19-Fällen nachgewiesen werden. Hervorgehoben wird Israel, mit einer vollständigen Impfrate von $60 \%$ der Gesamtbevölkerung und den meisten neu aufgetretenen COVID-19-Fällen. Andere Länder, wie z.B. Island und Portugal, wiesen bei noch höheren Impfquoten mehr nachgewiesene COVID-19-Fälle als Vietnam oder Südafrika auf, wo der Anteil an vollständig Geimpften bei ca. $10 \%$ lag. Ähnlich liegt der Fall bei der Analyse der US-Landkreise: Von den 5 Landkreisen mit dem höchsten Anteil an vollständig Geimpften wurden von den Centers for Disease Control and Prevention (CDC) 4 als Gebiete mit hoher Übertragungsrate identifiziert. Umgekehrt wiesen von den 57 Landkreisen, welche von den CDC als Landkreise mit "niedriger" Übertragungsrate identifiziert wurden, 15 (26,3\%) Impfraten von unter $20 \%$ auf. 
Diskussion. Aufgrund der Ergebnisse aus der Prä-Omikron Phase der Pandemie schließen die Autoren, dass das alleinige Vertrauen auf die Impfung als primäre Strategie im Kampf gegen COVID-19 überprüft werden müsse. Die Stigmatisierung der Bevölkerung könne mehr schaden als nutzen. Sie fordern, ergänzend andere Maßnahmen (z.B. Basishygiene, höhere Testfrequenz bzw. billigere Testmöglichkeiten) einzusetzen.

\section{Kommentar}

Im Folgenden soll auf vier schwerwiegende Limitationen in Bezug auf das Design bzw. die Schlussfolgerungen der Studie hingewiesen werden:

Erstens wird keine Auskunft darüber erteilt, wie lange die jeweils vollständige Impfung zurücklag. Je länger der Zeitraum zwischen Erfassung der COVID-19Fälle und vollständiger Impfung liegt, desto eher ist mit Impfdurchbrüchen zu rechnen. Dies hatte mit hoher Wahrscheinlichkeit einen Effekt auf das Auftreten von Impfdurchbrüchen und somit auch auf die Anzahl der COVID-19-Fälle.

Zweitens geben die Autoren keine Auskunft über die jeweilige Testhäufigkeit in den untersuchten Gebieten. Die Testhäufigkeit kann sich - je nach lokalen, politischen sowie wirtschaftlichen Voraussetzungen - in den verschiedenen Gebieten unterscheiden. Es ist davon auszugehen, dass dies auch Auswirkungen auf den Anteil der positiv Getesteten hat: in einem Gebiet mit höherer Testhäufigkeit ist mit einer höheren Fallzahl als in Gebieten mit niedrigerer Testhäufigkeit zu rechnen.

Drittens unterscheiden die Autoren nicht zwischen bevölkerungsreichen und ländlichen Gebieten. Es hat sich gezeigt, dass sich die Übertragungsdynamik in ländlichen Bezirken von jener in städtischen Bezirken unterscheidet [2] - dies könnte die Ergebnisse der Studie beeinflusst haben. Entsprechend konnte eine andere Arbeit, welche die 112 bevölkerungsreichsten Bezirke der USA einschloss (44,4\% der US-Gesamtbevölkerung), einen Zusammenhang von höheren Impfraten mit einer geringeren COVID-19Inzidenz sowie einer Reduktion schwerer COVID-19-Fälle nachweisen [3]. Diese Analyse umfasste auch demografische
Kovariablen - im Gegensatz zur Arbeit von Subramanian und Kumar.

Und viertens wird die Tatsache, dass die Impfung vor schwerem Krankheitsverlauf und Tod schützt, nicht annähernd auf einem akzeptablen Niveau diskutiert. Hier verweisen die Autoren auf Daten des israelischen Gesundheitsministeriums und behaupten, dass die Wirksamkeit des Impfstoffs BNT162b2 (Pfizer-BioNTech) gegen das Auftreten einer COVID19-Infektion nur $39 \%$ betrug. Tatsächlich zeigt dieselbe Quelle eine Wirksamkeit von $88 \%$ gegen COVID-19-Hospitalisierung sowie $91,4 \%$ gegen einen schweren COVID-19-Verlauf - einschließlich invasiver Beatmung, Schock und Tod [4]. Dieser Umstand wird von den Autoren nicht erwähnt.

Des Weiteren wird ein Auszug aus einem Bericht der CDC aus den USA zitiert, der einen Anstieg der Hospitalisierungsrate (von 0,01 auf 9\%) sowie der Sterberate im Krankenhaus (von 0 auf 15,1\%) zwischen Januar und Mai 2021 bei vollständig Geimpften zeigt [5]. Subramanian und Kumar stellen aufgrund dieser Zahlen die Impfwirksamkeit infrage. Jedoch führt derselbe Bericht diese Zahlen eindeutig auf einen im gleichen Zeitraum angestiegenen Anteil an vollständig Geimpften in der Gesamtbevölkerung zurück [5]; bemerkenswerterweise wird auch dieser Umstand von den Autoren nicht erwähnt. Außerdem gibt der zitierte CDC-Bericht selbst die Wirksamkeit von zwei Dosen eines mRNA-Impfstoffs mit $87 \%$ (gegen Hospitalisierung) bzw. $90 \%$ (gegen symptomatische Infektionen) an [5].

Darüber hinaus zeigte eine kürzlich veröffentlichte Fall-Kontroll-Analyse mit 4513 erwachsenen hospitalisierten Patienten, dass eine Hospitalisierung wegen COVID-19 signifikant mit einer geringeren Wahrscheinlichkeit einer Impfung assoziiert war (15,8\% vs. 54,8\%) [6]; in der gleichen Studie wurde bei 1197 mit COVID-19 hospitalisierten Patienten nachgewiesen, dass Tod oder invasive Beatmung mit einer geringeren Impfwahrscheinlichkeit einhergeht $(12,0 \%$ vs. 24,7\%) [6]. Die Autoren dieser Arbeit fanden auch einen höheren Anteil an Krankenhausentlassungen am Tag 28 bei Fällen mit Impfdurchbruch als bei ungeimpften Fällen (adjustierte „hazard ratio“
1,73; 95\%-KI 1,42-2,1). Dieses Ergebnis blieb auch nach Bereinigung nach Alter bzw. Beeinträchtigung des Immunsystems konsistent [6]. Dies weist darauf hin, dass eine Impfung nicht nur "Individuen gegen schwere Hospitalisation und Tod Schutz anbietet“ (Orig: „(...) vaccinations offers [sic!] protection to individuals against severe hospitalisation and death $\left.(\ldots)^{\prime \prime}\right)$ [1], sondern unabhängig von Alter bzw. Beeinträchtigung des Immunsystems sinnvoll ist.

\section{Fazit für die Praxis}

- In der Studie von Subramanian und Kumar wird der vielfach nachgewiesene Schutz durch Impfung gegen Coronavirus Disease 2019 (COVID-19) infrage gestellt. Sie ist ein Beispiel dafür, wie eine rein statistische Auswertung von Daten ohne deren kritische Betrachtung den eiligen oder unkritischen Leser zu fundamentalen Fehlschlüssen verleiten kann.

- Die Ergebnisse bzw. Schlussfolgerungen werden durch folgende Limitationen beeinflusst: Erstens erhält der Leser keine Informationen über den zeitlichen Abstand zwischen vollständigem Impfstatus und Erfassung der COVID-19-Fälle. Zweitens werden mögliche Unterschiede bezüglich der Testhäufigkeit zwischen den untersuchten Gebieten nicht adäquat diskutiert. Drittens könnte eine heterogene Studienpopulation bezüglich demografischer Faktoren und Bevölkerungsdichte die Ergebnisse der Studie von Subramanian und Kumar beeinflusst haben. Und viertens wird der Wert der Impfung inakzeptabel verkürzt und falsch dargestellt. Als Konsequenz erscheint die Schlussfolgerung des Artikels, dass „andere nichtpharmakologische Präventionsmaßnahmen (...) erneuert werden müssen, um die Balance zu finden, mit COVID 19 leben zu lernen (...)“ (Orig: "other non-pharmacological prevention (...) needs to be renewed in order to strike the balance of learning to live with COVID-19 (...) irreführend, da der Nutzen der lebensrettenden Impfung unterschätzt wird.

Korrespondenzadresse

Julian Umlauf

Klinik für Anästhesiologie und Intensivmedizin, Universitätsklinikum Augsburg

Stenglinstr. 2, 86156 Augsburg, Deutschland

julian.umlauf@uk-augsburg.de

Interessenkonflikt. J. Umlauf und A. R. Heller geben an, dass kein Interessenkonflikt besteht. 


\section{Literatur}

1. Subramanian SV, Kumar A (2021) Increases in COVID-19 are unrelated to levels of vaccination across 68 countries and 2947 counties in the United States. Eur J Epidemiol 36(12):1237-1240. https:// doi.org/10.1007/s10654-021-00808-7

2. LiY,HuT,GaiX,Zhang Y,ZhouX(2021)Transmission dynamics, heterogeneity and controllability of SARS-coV-2: a rural-urban comparison. Int J Environ Res Public Health 18:5221. https://doi.org/ 10.3390/ijerph18105221

3. Harris JE (2021) COVID-19 incidence and hospitalization during the delta surge were inversely related to vaccination coverage among the most populous U.S. Counties. Health Policy Technol. https://doi.org/10.1016/j.hlpt.2021.100583

4. Ministry of Health Israel (2021) Two-dose vaccination data. Government of Israel. https://www.gov.il/BlobFolder/reports/vaccineefficacy-safetyfollow-up-committee/he/files_ publications_corona_two-dose-vaccinationdata. pdf.Zugegriffen:22.12.2021

5. McMorrow M (2021) Improving communications around vaccine breakthrough and vaccine effectiveness. https://contextcdn.washingtonpost. com/notes/prod/default/documents/8a72640807bd-46bda945-3af0ae2f3c37/note/57c986043b54-44f0-8b44-b148d8f75165. Zugegriffen: 22.12.2021

6. Tenforde MW, Self WH, Adams K, Gaglani M, Ginde AA, McNeal T et al (2021) Association Between mRNA Vaccination and COVID-19 Hospitalization and Disease Severity. JAMA 326:2043-2054. https://doi.org/10.1001/jama. 2021.19499

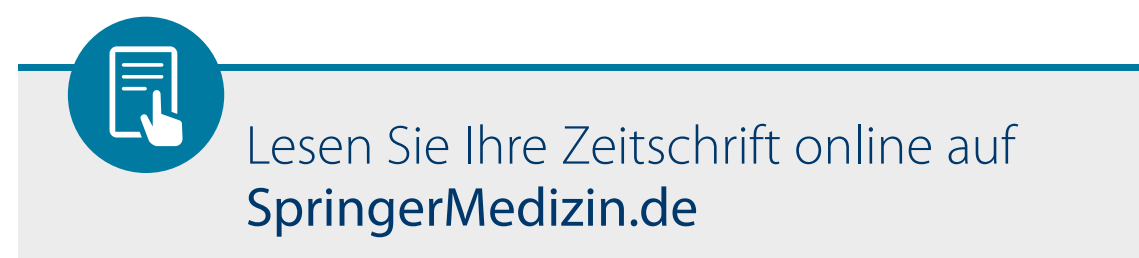

SpringerMedizin.de bietet lhnen Zugang zu allen elektronisch verfügbaren Ausgaben und dem CME-Angebot Ihrer Zeitschrift - unabhängig davon, seit wann Sie die Zeitschrift abonniert haben.

So einfach erhalten Sie Zugang zum Online-Archiv:

- Registrieren Sie sich einmalig auf www.springermedizin.de/register Geben Sie dabei Ihre Einheitliche Fortbildungsnummer (EFN) an.

- Ihr Benutzername entspricht Ihrer E-Mail-Adresse, Ihr Passwort können Sie frei wählen und später jederzeit unter "Mein Profil“ ändern.

- Falls Sie bereits ein (Print-) Abonnement bei uns haben, geben Sie bei der Registrierung die Lieferadresse Ihrer Zeitschrift an. Damit wird Ihr Abo-Zugang auf springermedizin.de freigeschaltet.

\section{Sind Sie bereits bei SpringerMedizin.de registriert?}

Dann wird Ihr Zeitschriftenabonnement automatisch Ihrem Online-Nutzerkonto hinzugefügt. Sollten die Angaben Ihres Online-Accounts nicht eindeutig mit den Angaben Ihres Zeitschriften-Abonnements übereinstimmen, kann die Zuordnung nicht sicher erfolgen. In diesem Fall und bei allen anderen Fragen zum Online-Zugang kontaktieren Sie bitte unseren Kundenservice unter: Kundenservice@springermedizin.de

Telefonisch erreichen Sie die Hotline montags bis freitags von 9.00 bis 17.00 Uhr kostenfrei unter 0800-77 80777 sowie gebührenpflichtig aus dem Ausland unter +4930884293600. 